\title{
The Official FENS Calendar
}

Ann Nutr Metab 2006;50:555

Published online: December 21, 2006

This section provides information about the meetings and events of all FENS member societies. The list of events is by no means inclusive, and the publisher is not responsible for any data given.

\begin{tabular}{|c|c|c|c|c|}
\hline Event & Place/Date & Organizing Society & Contact for information & Web-Site \\
\hline $\begin{array}{l}44^{\text {th }} \text { Scientific Congress of } \\
\text { the German Nutrition } \\
\text { Society: Nutrition - } \\
\text { Opportunities and Risks in } \\
\text { the } 21^{\text {st }} \text { Century }\end{array}$ & $\begin{array}{l}\text { Halle / Germany } \\
\text { March 8-9, } 2007\end{array}$ & German Nutrition Society (DGE) & $\begin{array}{l}\text { DGE } \\
\text { Department of Public Relations } \\
\text { Godesberger Allee } 18 \\
\text { D-53175 Bonn } \\
\text { Tel.: +49228 } 3776600 \\
\text { Fax: }+492283776800\end{array}$ & $\underline{\text { www.dge.de }}$ \\
\hline $\begin{array}{l}\text { Early Nutrition } \\
\text { Programming \& Health } \\
\text { Outcomes in Later Life: } \\
\text { Obesity \& Beyond }\end{array}$ & $\begin{array}{l}\text { Budapest / Hungary } \\
\text { April 20-21, } 2007\end{array}$ & $\begin{array}{l}\text { Early Nutrition Programming } \\
\text { Project / European Academy of } \\
\text { Nutritional Sciences (EANS) }\end{array}$ & $\begin{array}{l}\text { EARNEST } \\
\text { earnest@med.uni-muenchen.de }\end{array}$ & www.metabolic-programming.org \\
\hline $\begin{array}{l}10^{\text {th }} \text { European Nutrition } \\
\text { Conference }\end{array}$ & $\begin{array}{l}\text { Paris / France } \\
\text { July 10-13, } 2007\end{array}$ & $\begin{array}{l}\text { FENS / Union Française pour la } \\
\text { Nutrition et l'Alimentation (UFNA) }\end{array}$ & $\begin{array}{l}\text { BCA } \\
\text { 6, boulevard du Général Leclerc } \\
\text { F-92115 Clichy Cedex } \\
\text { e-mail: contact@b-c-a.fr }\end{array}$ & $\underline{w w w . b-c-a . f r}$ \\
\hline
\end{tabular}

